\title{
Papillary Carcinoma in a Thyroglossal Cyst: Report of a Case and Review of Literature
}

\author{
A. Thakar, Senior Resident \\ D. A. Tandon, Associate Professor \\ S. Sharma, Senior Resident \\ Department of Otorhinolaryngology and Head \& Neck Surgery; Pathology \\ All India Institute of Medical Science, New Delhi - 110029.
}

\section{Abstract}

A 29-years old male presenting with a midline neck swelling four centimetres in diameter of one years duration was diagnosed to have a thyroglossal cyst. He underwent a Sistrunk's operation. Histological examination of the specimen revealed a papillary thyroid carcinoma limited to the cyst wall. The thyroid scan was normal indicating no further need for surgery. The patient has been on suppressive therapy with thyroxin for one year and is currently disease-free. A review of literature is also preseirted.

\section{INTRODUCTION}

Dapillary carcinoma of the thyroglossal tract is rare. The diagnosis is usually made retrospectively following surgical excision of a supposedly benign thyroglossal cyst. For localized tumours Sistrunk's operation provides adequate surgical clearance. One such patient is reported and the management principles outlined.

\section{Case Report}

A 29-years old male presented with a midline swelling in the upper part of the neck, insidious in onset and of an year's duration. The swelling had rapidly increased in size over the past three months. There was no fever or weight loss. General physical examination was normal. Examination of the upper aerodigestive tract and indirect laryngoscopy were normal. A $4 \mathrm{~cm} \times 2 \mathrm{~cm}$ globular mass was present in the midline of the neck over the thyrohyoid space. It was non-tender, fluctuant and nonpulsatile. It demonstrated upward mobility on swallowing and on protrusion of the tongue. The thyroid lobes were normally palpable and there was no lymphadenopathy.

With a pre-operative diagnosis of a thyroglossal cyst the neck was explored via a horizontal neck incision. The cyst was found tethered to the midportion of the hyoid bone and a tract was found to lead inferiorly from the cyst to the thyroid isthmus. A Sistrunk's procedure was performed wherein the cyst, the body of hyoid, a cuff of central base tongue extending superiorly till the foramen caecum and the inferiorly extending tract were all removed in toto. Recovery was uneventful.

A section of the excised specimen revealed a cyst of $3.0 \mathrm{cms}$ diameter with a wall thickness of $0.2 \mathrm{cms}$. A solid area in one focus showed multiple small papillary projections $(0.5 \times 0.5$ $\mathrm{cms}$.). Sections from the solid area revealed a tumour with thin papillary projections with a fibrovascular core. The tumour cells were cuboidal with large ground glass nuclei showing grooving and pseudonucleoli at places. 
Psammoma bodies were also identified (fig. 1). The rest of the cyst wall was lined by flattened to squamous epithelium (fig. 2 \& 3). Thyroid tissue contained in the wall showed lymphocytic thyroiditis. A post-operative thyroid scan was normal. The patient has since been on suppressive therapy with thyroxin $0.1 \mathrm{mg}$ per day. He continues to be asymptomatic after one year.

\section{Discussion}

Only 85 cases of carcinoma in a thyroglossal remnant had been documented till 1979 (Batsakis, 1979). Since then, another forty have been reported in literature. The majority of these have been papillary carcinomas.

It was earlier hypothesized that differentiated thyroid tumours in association with the thyroglossal duct were due to metastasis or invasion from a primary thyroidal carcinoma (Judd, 1963). Subsequent detailed histological studies have however documented normal thyroid tissue in relation to the thyroglossal duct in $60-75 \%$ of specimens studied (Batsakis 1979; Livolsi et al 1974). Radiocative iodine scans have documented functioning thyroid tissue in relation to about a third of thyroglossal duct anomalies (Batsakis, 1979). Both these

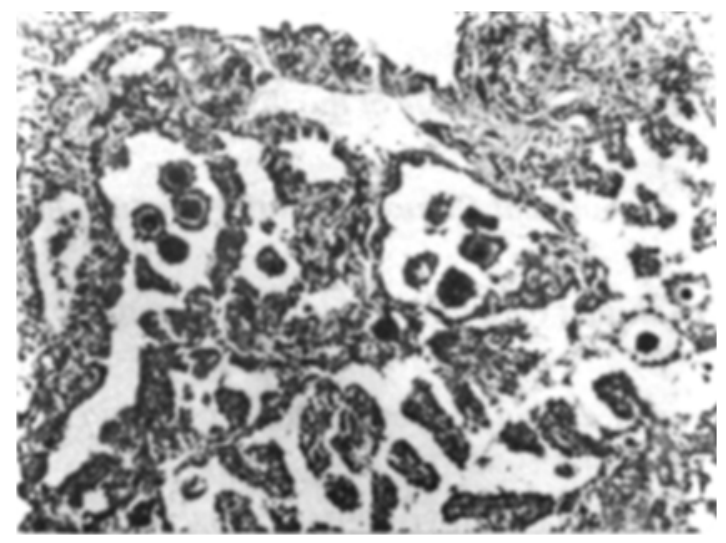

Fig 1. Shows the tumour to consist of papillae with a central fibrovascular core. Psammoma bodies are seen lying in the papillae as well as freely $(H \& E \times 20)$

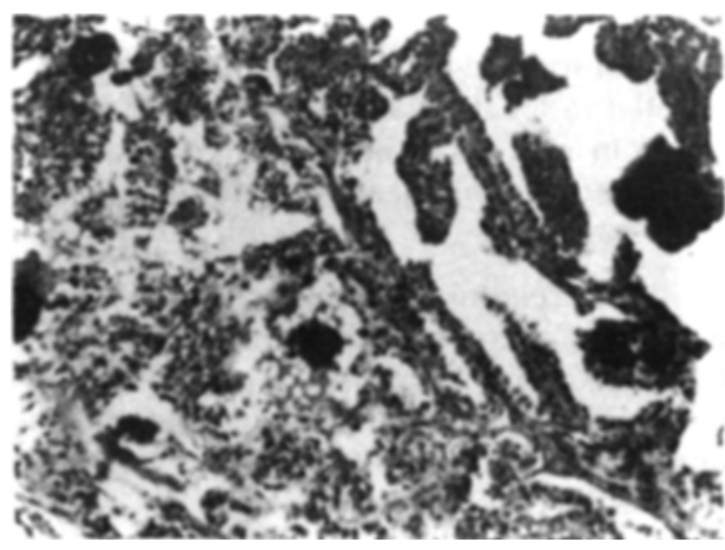

Fig 2. Shurns thi: p.pillae to be lined by a single layer of culiorlal cells with washed out nuclei $(H \& E \times 20)$.

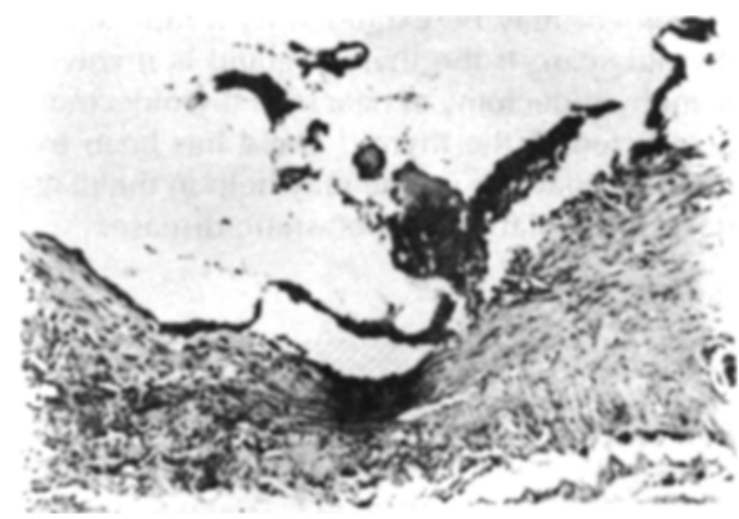

Fig 3. Tumour seen arising from the wall of a cyst lined by flattened cells (H\& Ex 10).

observations strongly suggest that thyroid carcinoma may arise de novo in thyroglossal remnants ( $k$ ristensen et al 1984). While papillary adenocarcinoma is the commonest $(75-80 \%)$, mixed papillary-follicular carcinomas also occur. Squamous cell carcinoma and anaplastic carcinoma have also been reported and are thought to arise from the lining of the thyroglossal duct.

The diagnosis of thyroglossal duct cardinoma is rarely made clinically. It occurs in less than $1 \%$ of thyroglossal duct anomalies. Apart from a painless, slowly enlarging midline mass it may occasionally manifest with pain, local fix- 
ity, dysphagia, hoarseness or rapid increase in size; but these signs are more likely to be attributed to local inflammation than to malignancy. In most instances diagnosis is made only after histoppathological evaluation of the surgical specimen.

Papillary crcinoma of the thyroglossal duct is not a very aggressive disease. The biological behavior is similar to that of papillary carcinoma of the thyroid gland. Nodal metastasis is uncommon and distant metastasis has been reported in only three patients.

Simultaneous involvement of the thyroid gland may occur by local metastasis or invasion of the thyroglossal duct by a primary thyroidal carcinoma or vice-versa. Thyroid gland involvement may be excluded by a radioactive thyroid scan. If the thyroid gland is involved hemithyroidectomy or near total thyroidectomy is indicted. If the thyroid gland has been excised radioactive iodine may help in the diagnosis and ablation of metastatic disease.
Papillry carcinoma of the thyroid is considered to be an endocrine dependent tumour, the growth of which is augmented with thyroid stimulating hormone (Crile, 1966). Exogenous thyroxin which acts by decreasing TSH secretion is therefore indicated in all patients to suppress microscopic residual or metastatic disease.

Sistrunk's operation by which the cyst, the central portion of the hyoid bone, the foramen caecum with a surrounding portion of the central base tongue and cuff of tissue from the cyst to the thyroid isthmus is removed has been considered adequate surgical clearance for a papillary carcinoma of the thyroglossal cyst demonstrating no invasion beyond the cyst wall (Kristensen et al 1984, Trail et al 1977). Careful long term follow-up is important as papillary carcinoma is usually a low grade malignancy and recurrences can generally be successfully handled by additional surgical resection in association with radio-iodine and external radiotherapy.

\section{Reference}

1. Batsakis JG (1979) Tumours of the Head and Neck: Clinical and Pathological Considerations. Second Edition. Williams and Wilkins, Baltimore, pp 237.

2. Crile JG (1966) Endocrine dependency of papillary car carcinomas of the thyroid. Journal of American Medical Association, 195: 721-724.

3. Judd ES (1963) Thyroglossal duct cyst and sinuses. Surgical Clinics of North America, 43: 1023-1032.

4. Kristensen S, Juul A, Moesner J (1984) Thyroglossal duct carcinoma. Journal of Laryngology and Otology, 98:1277-1280

5. Livolsi VA, Perzin KH, Savetsky L (1974) Carcinoma arising in Median Ectopic thyroid (including thyroglossal duct tissue). Cancer, 34: 1303-1315.

6. Trail ML, Zeringue GP, Chicola JP (1977) Carcinoma in thyroglossal duct remnants. Laryngoscope, 87: 1685-1691. 